\title{
Salud autopercibida, apoyo social y familiar de los pacientes con enfermedad pulmonar obstructiva crónica
}

\author{
A. M. Fernández Vargas, M. J. Bujalance Zafra, F. Leiva Fernández, F. Martos \\ CRESPO*, A. J. Garcia Ruiz*, F. SánChez de la Cuesta y AlarCón** \\ Dra. en Medicina y Cirugía. Especialista en Medicina Familiar y Comunitaria. \\ Unidad Docente de MFyC de Málaga. \\ *Profesor titular. Departamento de Farmacología y Terapéutica Clínica. Facultad de Medicina. \\ Universidad de Málaga. \\ **Catedrático. Departamento de Farmacología y Terapéutica Clínica. Facultad de Medicina. \\ Universidad de Málaga.
}

\section{RESUMEN}

Fundamentos: la EPOC es una enfermedad crónica e invalidante con gran repercusión en la calidad de vida del paciente así como en su diná mica familiar y social. La percepción por parte del paciente de sus limitaciones genera un sentimiento de baja autoestima que pone en peligro su posición ante la familia y la sociedad.

Objetivos: describir características sociodemo gráficas y conocer en los pacientes con EPOC la calidad de vida autopercibida, el apoyo social con que cuentan y su dinámica familiar.

Diseño: descriptivo transversal.

Material y métodos: selección mediante mues treo consecutivo de 278 pacientes diagnosticados de EPOC. Mediante entrevista personal se recogió: perfil sociodemográfico, hábito tabáquico y antece dentes clínicos (comorbilidad), salud autopercibida (perfil de salud de Nottingham, NHP, y Cuestiona rio Respiratorio St George, SGRQ), apoyo social (Escala de Duke-Unc) y apoyo familiar (test de AP GAR familiar). Estadística descriptiva.

Resultados: variables sociodemográficas: varo nes (88\%), de 66,9 $\pm 8,9$ años, casados (87\%), anal fabetos (46,7\%), jubilados (77\%). Fumadores (76\%) de 53,2 paquetes/año. Comorbilidad: $76 \%$ pacientes. Los principales resultados de salud au topercibida fueron (medias e IC 95\%): perfil de Salud de Nottingham (0-100): subescalas de ener gía 40 (35,6-44,4), dolor 35,9 (32,3-39,5), emotivi dad 32,5 (29,4-38,6), sueño 41,9 (37,8-45,9), so cial 15,3 (12,7-17,9), movilidad 36,7 (33,9-39,5) y total 33,4 (30,8-36). Cuestionario de St. George (0-100): subescalas de impacto 38,01 (35,0840,18), actividad 53,8 (50,2-57,4), sintoma 37,7 $(35,2-40,3)$ y total 40,9 $(38,6-43,2)$. Apoyo social
Quality of life, familiar and social network of patients with chronic obstructive pulmonary disease

\section{ABSTRACT}

Backgrounds: Chronic Obstructive Pulmonary Disease (COPD) is a chronic and invalidant ill ness with a great impact on quality of life, and fa mily and social dynamics. Patients perceive limita tions caused by their health status and this causes a low self-steem that threatens their position in their families and society

Aims: 1. describe sociodemographic characte ristics of patients with COPD. 2. Analize the qua lity of life, social network and family dynamics of these patients.

Design: cross-sectional study.

Participants: 278 patients with COPD (confi dence level 95\%) from two urban health centers.

Main measurements: personal interview. Varia bles: quality of life (Nottingham Health Profile; St George Respiratory Questionnaire); social net work (Duke-UNC scale); family dynamics (AP GAR family test);sociodemographic profile; smo king habit and comorbidity. Statistical analises: descriptive statistics.

Results: sociodemographic profile: age: $66.9 \pm 8,9$ years; sex: (88\%) male; married $(87 \%)$; illiterates (46.7\%); retired people (77\%); smoking habit (76\%) with a mean consum of 53.2 boxes per year. Comorbidity: $76 \%$. Quality of life scales (me an and standard deviation): Nottingham Health Profile subscales (total score 100 points): energy

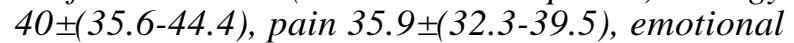
reactions 32.5 $\pm(29.4-38.6)$, sleep 41.9 $\pm(37.8$ 45.9), social isolation 15.3 $\pm(12.7-17.9)$, mobility $36.7 \pm(33.9-39.5)$, global score 33.4 $\pm(30.8-36)$. St George Respitatory Questionnaire subscales (total scores 100 points): impact 38.01 $\pm(35.08-40.18)$, ac tivity $53.8 \pm(50.2-57.4)$, symptoms $37.7 \pm(35.2-40.3)$ 
afectivo bueno: $65,1 \%$ de pacientes, apoyo social confidencial escaso: $57,4 \%$ de pacientes. APGAR familiar: normal 79,7\%, disfunción moderada $17,4 \%$ y disfunción grave $2,9 \%$.

Conclusiones: la calidad de vida de los pacien tes con EPOC resultó estar bastante alterada fun damentalmente en las dimensiones de sueño, ener gía y movilidad del NHP y en la subescala que valora la limitación de la actividad a causa de la disnea del SGRQ. Algo más de la mitad de los pacientes referían buen apoyo social, siendo mayor el afectivo que el confidencial. Consideramos impor tante el porcentaje de disfunción familiar (20,3\%).

Palabras clave: Enfermedad Pulmonar Obs tructiva Crónica. Calidad de vida. Apoyo social y familiar

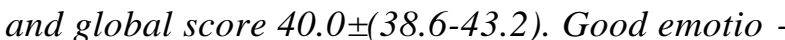
nal social support (61\% patients); limited confi dential social support in $57.4 \%$ patients. APGAR test: mild family disfunction in $17.4 \%$ and severe family disfunction in $2.9 \%$.

Conclusions: quality of life in COPD patients is affected in de following dimensions: sleep, energy and mobility assessed by NHP and in the subscale activity assessed by the SGRQ. Abit more than a half of patients referred to have an acceptable so cial support (the emotional support is greater than the confidential one). We have found a considera ble percentage of family disfunction.

Key words: Chronic Obstructive Pulmonary Disease. Quality of life. Sociofamily support.

\section{INTRODUCCIÓN}

La EPOC es una de las enfermedades respiratorias crónicas más frecuentes y representa un problema de salud con importantes implicaciones sociosanitarias, debido a la elevada morbimortalidad que conlleva. Tanto en España como en Europa, son escasos los datos sobre morbilidad específica por EPOC. Sin embargo, las encuestas periódicas de morbilidad hospitalaria muestran tendencia al alza en el número de ingresos por esta causa y se calculan unos 25.000 días de ingreso hospitalario por cada millón de habitantes. Entre 30 y 120 individuos por cada 100.000 habitantes reciben oxigenoterapia domiciliaria, casi siempre por EPOC ${ }^{1,2}$. Además hay que considerar la pérdida de días de trabajo que origina y la demanda de apoyo y ayudas sociales que produce. En cuanto a mortalidad en España, supone la $5^{\mathrm{a}}$ causa de muerte en varones (tasa de 53,9/10.000 hab 1997) y la $7^{\mathrm{a}}$ en las mujeres (tasa 12,4/10.000 hab 1997) ${ }^{3}$.

Esta enfermedad genera en el paciente una pérdida progresiva de la capacidad funcional hasta limitarlo en las actividades más simples de la vida cotidiana, lo que repercute en su calidad de vida.

Es importante conocer la valoración personal que el individuo realiza acerca de cómo la enfermedad y el tratamiento correspondiente repercuten sobre su estilo de vida, de acuerdo con su propia percepción e interpretación. Esta faceta subjetiva de la morbilidad (gravedad percibida por el paciente) es lo que definimos como calidad de vida relacionada con la salud (CVRS).

La percepción por parte del sujeto de sus limitaciones genera un sentimiento de baja autoestima que pone en peligro su posición ante la familia y la sociedad, lo que puede producir por un lado, una disfunción familiar entendida como aquel proceso que impide cumplir sus funciones a la familia, de acuerdo con la etapa del ciclo vital en que se encuentra y en relación con las demandas que percibe de su entorno. Y por otro, una alteración en las relaciones sociales del paciente. Sin embargo, el tener un buen apoyo social, entendido como el grado en el que las necesidades sociales básicas son satisfechas, puede favorecer el manejo de la intervención preventiva, terapéutica y rehabilitadora de distintos problemas de salud, mediante el fortalecimiento de las relaciones intrafamiliares o el refuerzo de grupos de autoayuda. Tanto la salud física como la psíquica mejoran al aumentar el apoyo social.

Por todo ello, nos planteamos realizar un trabajo que nos ayude a conocer "quiénes son" los pacientes que sufren esta enfermedad, y "cómo se encuentran desde el punto de vista psicosocial", valorando para ello, su calidad de vida, su apoyo familiar y social.

\section{SUJETOS Y MÉTODOS}

Se realizó un estudio descriptivo transversal en dos centros de salud urbanos El Palo y La PalmaPalmilla, que contaban con programa de crónicos y subprograma de EPOC en desarrollo en los últimos años.

El centro de salud El Palo atiende a una población de 34.660 habitantes, con varios núcleos rurales y semirrurales. La pirámide de población sigue un proceso de inversión, de base estrecha y cúspide ancha (población mayor de 65 años 10,8\%). El nivel socioeconómico es heterogéneo, y en general predomina el medio bajo (tasa de paro $=30 \%$, tasa de analfabetismo $=13,7 \%)$. En el ámbito de las relaciones sociales son barrios de fuerte raigambre y 
tradición de relaciones vecinales estrechas, en las que el sentido de pertenencia refuerza al individuo con problemas de salud, y los vecinos se convierten en una importante fuente de apoyo social.

El centro de salud de La Palmilla atiende a una población de 24.585 habitantes. La pirámide de población se caracteriza por ser de tipo regresivo (bulbo): base estrecha que se ensancha hacia el centro, para estrecharse nuevamente en el vértice, siendo el grupo poblaciones más numeroso entre 25-39 años sin apenas diferencias entre sexos. Los mayores de 65 años suponen el $5 \%$ del total de población. El nivel socioeconómico es bajo (tasa de paro: $60 \%$, tasa de analfabetismo entre 10-14\%). Se considera zona marginal y de alto riesgo social.

La elección de este ámbito, puede producir un sesgo de selección, sin embargo proporciona mayor accesibilidad a los registros que por otra parte recogen información protocolizada y de calidad, aunque puedan modificarse los datos de prevalencia de la EPOC que no es objetivo de nuestro trabajo. Se seleccionaron de forma consecutiva del registro general de población dependiente de cada centro de salud (población atendida con historia clínica abierta en el centro de salud), a pacientes de ambos sexos con patología respiratoria, distinguidos mediante código informatizado (Centro de Salud La Palmilla) o pegatina verde (Centro de Salud El Palo).

Se incluyeron a los pacientes mayores de 35 años, pues la EPOC es una enfermedad que se manifiesta en pacientes de edad avanzada ${ }^{4-7}$ como mínimo en la edad media de la vida, "diagnosticados previamente de EPOC" por los distintos facultativos que les atendían y que contaban con historia clínica en los centros de salud. Se excluyeron a los pacientes que presentaban obstrucción crónica al flujo aéreo con diagnósticos específicos como bronquiectasias, fibrosis quística y asma, entidades explícitamente excluidas por la Sociedad Española de Neumología y Cirugía Torácica (SEPAR) ${ }^{5}$ y por la American Thoracic Society (ATS) ${ }^{8}$ de la definición de EPOC, y aquellos pacientes que aún cumpliendo los criterios de inclusión no se encontraban en la zona básica de salud (ZBS) en el momento del estudio (localizados telefónicamente pero desplazados por distintos motivos).

Se consideró paciente diagnosticado de EPOC cuando aparecía de forma explícita este diagnóstico en la historia clínica de los pacientes.

La población total con criterios de inclusión fue de 658 sujetos. Se accedió a la historia clínica personal de cada uno de estos pacientes de donde se recogieron algunas de las variables del estudio y un teléfono de contacto. Posteriormente se procedió a la citación de los pacientes mediante llamada telefónica (5 como mínimo), informándoles de las características del estudio en cuanto a voluntariedad en la participación, confidencialidad de los datos y objetivo del mismo. Los pacientes que no tenían teléfono o que no se localizaron tras 5 llamadas a diferentes horas y días se consideraron "pacientes perdidos", ya que necesitábamos que el paciente acudiera al centro de salud para cumplimentar los cuestionarios de calidad de vida, apoyo social y dinámica familiar. La entrevista personal tenía lugar en los respectivos centros de salud con un tiempo promedio total de aproximadamente 40 minutos.

El estudio se realizó sobre los sujetos registrados a fecha de 15 de diciembre de 1998. Los datos correspondientes a las variables de estudio se recogieron desde 18 de enero a 11 de junio de 1999 .

Las variables recogidas objeto de este estudio fueron:

-Datos sociodemográficos del paciente: edad (años), sexo, peso $(\mathrm{kg})$, talla $(\mathrm{cm})$, índice de masa corporal (IMC), estado civil, nivel de estudios, situación laboral.

- Factores de riesgo para la EPOC: hábito tabáquico, se clasificó en no fumador (nunca había fumado), fumador activo (sí fumaba cualquier cantidad de cigarrillos diariamente en el último mes) y exfumador (más de 6 meses sin fumar). Se cuantificó asimismo, el número de paquetes/año (número de cigarrillos fumados cada día, dividido por 20 cigarrillos que tienen cada paquete y multiplicados por el número de años fumando). Además se identificó, según el tipo de trabajo realizado, el riesgo de exposición laboral: riesgo físico (si existía exposición a la humedad, frío, lluvia, etc., en trabajos a la intemperie como pescadores, jardineros, mantenimiento de calles, trabajadores de la construcción); riesgo de exposición a determinados productos (en fábricas de cemento, minas, pintores, curtidores de pieles, carpinteros, panaderos) y sin riesgo (vendedores, hostelería y servicios, oficinas).

-Antecedentes patológicos: psicopatologías (ansiedad, depresión, insomnio) por los efectos de los mismos y su tratamiento en el curso clínico de la EPOC; osteoarticulares (artrosis y artritis) por la limitación de la movilidad que puede generar; hipertensión arterial (HTA), dislipemias y diabetes por la frecuencia de estas patologías, y trastornos cardiovasculares (cardiopatía isquémica, arteriopatía arterioesclerótica, aneurisma, insuficiencia cardiaca, insuficiencia venosa, fibrilación auricular, valvulopatía), por su poder de enmascaramiento de la disnea respiratoria.

- Salud autopercibida: utilizamos 2 cuestionarios: uno genérico, el Perfil de Salud de Nottingham (NHP) ${ }^{9}$ que consta de 38 ítems pertenecientes a 6 dimensiones: energía, dolor, emotividad, sueño, aislamiento social y movilidad física. Se completa con 7 preguntas sobre limitaciones a causa de la salud en 7 actividades de la vida diaria. Y otro espe- 
cífico de patología respiratoria, el Cuestionario respiratorio de St. George (SGRQ) $)^{10}$ que consta de 50 ítems repartidos en 3 escalas: síntomas (frecuencia y severidad de los síntomas respiratorios), actividad (limitación de la actividad a causa de la disnea) e impacto (alteraciones psicológicas y de funcionalismo social producidas por la enfermedad respiratoria). Ambos cuestionarios han sido validados y traducidos al español y se puntúan de 0 (mejor estado de salud) a 100 (peor estado).

-Apoyo social, mediante la escala de DUKE$\mathrm{UNC}^{11}$, consta de 11 ítems que recogen valores referidos al apoyo afectivo y confidencial. El apoyo confidencial se valora a través de 5 ítems que se corresponden con las preguntas 2, 3, 5 ,9 y $11 \mathrm{del}$ cuestionario, con puntuación total de 25 . Se considera apoyo confidencial bueno si obtenemos más de 15 puntos en este apartado. Para valorar el apoyo afectivo contamos con 6 ítems que se corresponde con las preguntas $1,4,6,7,8$, y 10 del test, con puntuación total de 30 . Si se obtiene más de 18 puntos habrá buen apoyo afectivo.

-Dinámica familiar mediante el Test de APGAR $^{12}$, consta de 5 preguntas que mide los componentes del funcionalismo familiar en cuanto a adaptabilidad, cooperación, desarrollo, afectividad y capacidad resolutiva. Cada pregunta se puntúa de 0 a 2 puntos. Se considera buena función familiar si se obtiene entre 7 y 10 puntos, moderada disfunción si el resultado es de 4 a 6 puntos y disfunción grave si la puntuación es menor de 4 .

Se realizó un análisis descriptivo de todas las variables contempladas en el estudio, calculando como estadístico de tendencia central la media y mediana, y de dispersión la desviación estándar para variables cuantitativas. Para variables cualitativas se han calculado las frecuencias absolutas y relativas de cada categoría. Se calculan los intervalos de confianza al $95 \%$ para una media y una proporción. El análisis de los datos se realizó mediante el paquete estadístico SPSS/PC versión 8.0.

\section{RESULTADOS}

La participación real fue de 278 pacientes. La no localización telefónica (319 pacientes) constituyó la principal causa de no respuesta de los pacientes con criterios de inclusión. Otro grupo de no respuesta fueron los pacientes incapacitados por ingreso hospitalario o por encontrarse en su domicilio con incapacidad para desplazarse al centro de salud, debido a su propia patología respiratoria o a la patología concomitante (11 pacientes).

El fallecimiento de algunos de los pacientes incluidos en el estudio justifica una parte de las pérdidas (10 pacientes). Finalmente se negaron a la participación de forma explícita o por faltar reiteradamente a la cita 40 pacientes.

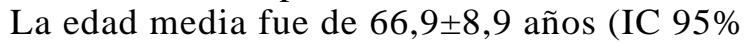
65,8-67,9). El 75\% tenían una edad comprendida entre 60 y 79 años. La distribución por género fue de 244 varones y 34 mujeres, con claro predominio del género masculino ( $88 \%)$. En cuanto al estado civil, 242 pacientes estaban casados, 19 viudos, 12 solteros y 5 separados. El nivel de estudios fue bajo, el 46,7\% no sabía leer ni escribir. Respecto a la situación laboral, la mayoría fueron jubilados $(77,1 \%)$. El peso medio fue de $77,6 \pm 14,4 \mathrm{~kg}$, la talla media fue de $165 \pm 7,9 \mathrm{~cm}$ y el IMC de $28,4 \pm 5$ $\mathrm{kg} / \mathrm{m}^{2}$ (IC 95\%; 27,8-28,9). El 41,7\% de los pacientes tuvieron un IMC entre 25-30 lo que indica sobrepeso tabla I.

El 24,5\% de los pacientes habían tenido exposición a determinados contaminantes de tipo laboral fundamentalmente trabajadores en fábricas de cemento, pintores, carpinteros, curtidores, etc., frente al $42,4 \%$ que no habían tenido ningún riesgo de exposición laboral.

De los 278 pacientes del estudio, 212 (76\%) habían sido fumadores, con una media de 53,2 paquetes/año (mediana $=52 ; \mathrm{DS}=32,4)$. De ellos, 150 (70,7\%) continuaban con el hábito tóxico, con una media de 61 paquetes/año (mediana=57; IC $95 \%=55,9-66,8)$.

El $76 \%$ de los pacientes presentaron una o más patologías concomitantes siendo la más prevalente la hipertensión con un 51,8\%, seguida de patología cardiovascular, trastornos osteoarticulares, diabetes, trastornos del metabolismo lipídico y psicopatologías (Tabla II).

Los resultados de calidad de vida relacionada con la salud obtenidos mediante el NHP reflejan puntuaciones medias más altas (indicador de peor estado de salud) en todas las subescalas, excepto en la de aislamiento social, destacando las áreas que miden sueño $(41,9)$, energía (40) y movilidad $(36,7)$. La puntuación media del NHP total fue de 33,4 (Tabla III).

Con el cuestionario específico de patología respiratoria utilizado la puntuación media total fue más alta que la obtenida con el perfil de Nottingham, 40.9 frente a 33.4, respectivamente. La calidad de vida del paciente EPOC se ve más afectada en la dimensión que valora la limitación de la actividad a causa de la disnea (53.8 de puntuación media) (Tabla 3).

Con respecto al apoyo social, la puntuación media del apoyo social afectivo fue de 16,6 (bueno $>18$ puntos) y del apoyo social confidencial fue de 19,2 (bueno > 15 puntos). El 57\% de los pacientes referían tener un buen apoyo social de forma global ( >33 puntos), siendo mayor el porcentaje de pacientes que presentaban un buen apoyo afectivo (65\%) que el confidencial $(47,5 \%)$ (Tabla IV). 
CARACTERÍSTICAS SOCIODEMOGRÁFICAS DE LOS PACIENTES DEL ESTUDIO. IC: INTERVALO DE CONFIANZA AL 95\%

\begin{tabular}{|c|c|c|c|c|}
\hline \multicolumn{2}{|c|}{ Variables sociodemográficas } & \multirow{2}{*}{$\begin{array}{c}\text { Frecuencia } \\
(n=278)\end{array}$} & \multirow{2}{*}{$\begin{array}{c}\% \\
1,4 \\
1,5 \\
14,5 \\
44,5 \\
31,6 \\
6,5\end{array}$} & \multirow{2}{*}{$\begin{array}{c}\text { IC } 95 \% \\
0,25-12,9 \\
0,25-12,0 \\
3,5-25,2 \\
35,8-53,3 \\
21,9-41,3 \\
4,8-17,8\end{array}$} \\
\hline Edad & $\begin{array}{l}\text { Menos de } 41 \\
41-49 \text { años } \\
50-59 \text { años } \\
60-69 \text { años } \\
70-79 \text { años } \\
>80 \text { años }\end{array}$ & & & \\
\hline Género & $\begin{array}{l}\text { Varón } \\
\text { Mujer }\end{array}$ & $\begin{array}{c}244 \\
34\end{array}$ & $\begin{array}{l}87,8 \\
12,2\end{array}$ & $\begin{array}{c}83,7-91,9 \\
1,1-23,2\end{array}$ \\
\hline IMC & $\begin{array}{l}<20 \\
20-25 \\
25-30 \\
>30\end{array}$ & $\begin{array}{c}5 \\
67 \\
116 \\
90\end{array}$ & $\begin{array}{c}1,8 \\
24,1 \\
41,7 \\
32,4\end{array}$ & $\begin{array}{r}-9,8-13,4 \\
13,8-34,3 \\
32,7-50,6 \\
22,7-42,6\end{array}$ \\
\hline Estado civil & $\begin{array}{l}\text { Soltero } \\
\text { Casado } \\
\text { Separado } \\
\text { Viudo }\end{array}$ & $\begin{array}{c}12 \\
242 \\
5 \\
19\end{array}$ & $\begin{array}{c}4,3 \\
87,1 \\
1,8 \\
6,8\end{array}$ & $\begin{array}{c}-7,1-15,7 \\
82,8-91,3 \\
1,1-2,4 \\
4,5-18,1\end{array}$ \\
\hline Nivel estudios & $\begin{array}{l}\text { Sin estudios } \\
\text { Sabe leer y escribir } \\
\text { Primarios } \\
\text { Bachiller } \\
\text { Superiores } \\
\text { Otros* }\end{array}$ & $\begin{array}{l}128 \\
43 \\
84 \\
12 \\
6 \\
1\end{array}$ & $\begin{array}{l}46,7 \\
15,7 \\
30,7 \\
4,4 \\
2,2 \\
0,4\end{array}$ & $\begin{array}{c}38-55,3 \\
4,9-26,5 \\
20,8-40,5 \\
-7,2-16 \\
-9,5-13,9 \\
-11,9-12,7\end{array}$ \\
\hline Situación laboral & $\begin{array}{l}\text { Trabaja } \\
\text { En paro } \\
\text { Jubilado } \\
\text { Otros** }\end{array}$ & $\begin{array}{c}17 \\
11 \\
212 \\
35\end{array}$ & $\begin{array}{c}6,2 \\
4,0 \\
77,1 \\
12,7\end{array}$ & $\begin{array}{c}5,2-17,6 \\
-7,5-15 \\
71,4-82,5 \\
1,6-23,7\end{array}$ \\
\hline
\end{tabular}

Nivel de estudios Otros*: casos perdidos. Situación laboral Otros**: trabajo no remunerado (ama de casa, voluntario).

Finalmente, el APGAR familiar valoró que el $79,7 \%$ de los pacientes tenían una función familiar normal, el 17,4\% presentaban una disfunción familiar moderada y el 2,9\% una disfunción familiar grave (Tabla IV).

\section{DISCUSIÓN}

En este estudio hemos tenido una elevada tasa de no respuesta, cuya causa principal fue la no localización telefónica del paciente como consecuencia fundamentalmente de cambios en el número de teléfono (127 casos), no registro del mismo en la historia clínica de los pacientes, o que no contestaron después de realizar al menos 5 llamadas a distintas horas del día y en distintos días. Este hecho puede ser debido a una escasa actualización de los registros o a falta de notificación por parte de los pacientes.
En general pensamos que, dados los motivos de pérdidas antes comentados, es posible que hayan quedado fuera del estudio pacientes con estadios más avanzados de su proceso, o sujetos de mayor edad con pérdida de su autonomía (lo que justificaría su estado de incapacidad o su desplazamiento fuera de la zona básica), por lo que nuestros resultados podrían estar magnificados.

La mayoría de nuestros pacientes fueron varones (88\%) con una edad media de 69,9 lo que coincide con los resultados de otros estudios realizados en nuestro medio ${ }^{4,13-16}$. En este hecho puede influir el mayor consumo de tabaco en los hombres, así como el mayor consumo de alcohol y mayor riesgo de exposición laboral, factores de riesgo que se van acumulando a lo largo de la vida. En las poblaciones donde se ha identificado un hábito similar entre ambos sexos no existe diferencia en la prevalencia de la EPOC ${ }^{17}$. En nuestro estudio encontramos una 
FACTORES DE RIESGO Y CARACTERÍSTICAS CLÍNICAS DE LOS PACIENTES DE ESTUDIO

Factores de riesgo y características

clínicas

Frecuencia

$(n=278)$

\begin{tabular}{llccc}
\hline Tabaco & No han fumado nunca & 66 & 23,7 & $14-34$ \\
& Sí han fumado alguna vez. & $212 \#$ & 76,3 & $70-81$ \\
\hline Fumador & Activo* & $150(n=212) \#$ & $70,75 \%$ & $63,8-76,1$ \\
& Exfumador* & $62(\mathrm{n}=212) \#$ & $29,24 \%$ & $22,8-35,1$ \\
\hline \multirow{2}{*}{ Riesgo laboral } & Riesgo físico & 91 & 32,7 & $23,1-42,3$ \\
& Riesgo de expocición ${ }^{2}$ & 68 & 24,5 & $14,3-34,6$ \\
& Sin riesgo & 118 & 42,4 & $33,6-51,2$ \\
\hline Comorbilidad & Hipertensión & 144 & 51,8 & $43,6-59,9$ \\
& Cardiovascular & 95 & 34,2 & $24,7-43,7$ \\
& Osteoarticular & 92 & 33,1 & $25,5-42,6$ \\
& Diabetes & 70 & 25,2 & $15-35,3$ \\
& Hipercolesterolemia & 61 & 21,9 & $11,5-32,2$ \\
& Hipertrigliceridemia & 50 & 18 & $7,3-28,6$ \\
& Dislipemia mixta*** & 42 & 15,1 & $4,4-25,9$ \\
& Psicopatología & 42 & 15,8 & $5-26,5$ \\
\hline
\end{tabular}

Fumador activo*: si fuma cualquier cantidad de cigarrillos diariamente en el último mes.

Exfumador**: más de 6 meses sin fumar.

Dislipemia mixta***: presencia de hipercolesterolemia más hipertrigliceridemia.

Riesgo físico ${ }^{1}$ : si existía exposición a la humedad, frío, lluvia, etc., en trabajos a la intemperie como pescadores, jardineros, mantenimiento de calles, trabajadores de la construcción.

Riesgo de exposición ${ }^{2}$ a determinados productos (en fábricas de cemento, minas, pintores, curtidores de pieles, carpinteros, panaderos). Sin riesgo (vendedores, hostelería y servicios, oficinas).

\section{Tabla III}

PUNTUACIONES DE CALIDAD DE VIDA EN LOS PACIENTES CON EPOC OBTENIDA CON EL PERFIL DE SALUD DE NOTTINGHAM (NHP) Y CON EL CUESTIONARIO RESPIRATORIO ST. GEORGE (SGRQ). VALORES DE LAS ESCALAS DE 0-100

\begin{tabular}{lllll}
\hline NHP & Media & Mediana & Desv. St & IC $95 \%$ \\
\hline Energía & 40 & 33,3 & 36,9 & $35,6-44,4$ \\
Dolor & 35,9 & 25 & 31,4 & $32,3-39,5$ \\
Emotividad & 32,5 & 33,3 & 27,1 & $29,4-38,6$ \\
Sueño & 41,9 & 40 & 34 & $37,8-45,9$ \\
A. Social & 15,3 & 0 & 22,1 & $12,7-17,9$ \\
Movilidad & 36,7 & 37,5 & 24,5 & $33,9-39,5$ \\
NHP total & 33,4 & 30,6 & 21,8 & $30,8-36$ \\
& & & & IC $95 \%$ \\
SGRQ & Media & Mediana & Desv. St & $35,2-40,3$ \\
\hline Síntoma & 37,7 & 36,7 & 21,3 & $50,2-57,4$ \\
Actividad & 53,8 & 55,0 & 30,4 & $35,8-40,1$ \\
Impacto & 38,0 & 34,3 & 18,2 & $38,6-43,2$ \\
Total & 40,9 & 38,8 & 19,0 & \\
\hline
\end{tabular}

proporción de varones fumadores del $88,2 \%$ frente a las mujeres del $11,8 \%$.

El 24,5\% de los pacientes habían tenido exposición a determinados polvos y productos químicos como consecuencia de su actividad laboral (elevado número de pintores entre los pacientes) $\mathrm{y}$, aunque este factor se ha relacionado con un mayor riesgo de padecer $\mathrm{EPOC}^{18}$, es difícil aislar su efecto 
Tabla IV

APOYO SOCIAL Y APGAR FAMILIAR EN LOS PACIENTES CON EPOC

\begin{tabular}{lllll} 
Escala de DUKE & Media & Mediana & Desv. St & IC $95 \%$ \\
\hline $\begin{array}{l}\text { APOYOAFE } \\
\text { Rango: } 6-30(18)^{*}\end{array}$ & 16,6 & 17 & 3,19 & $16,2-16,9$ \\
\hline $\begin{array}{l}\text { APOYOCON } \\
\text { Rango: } 5-25(15)^{* *}\end{array}$ & 19,22 & 18 & 5,6 & $18,5-19,8$ \\
\hline $\begin{array}{l}\text { APOYOSOC } \\
\text { Rango: } 11-55(33) \#\end{array}$ & 35,7 & 35 & 8,3 & $34,7-36,6$ \\
\hline
\end{tabular}

\begin{tabular}{lllll} 
APGAR familiar & Media & Mediana & Desv. St & IC $95 \%$ \\
\hline APGARTOT $^{\&}$ & 7,9 & 8 & 2,1 & $7,6-8,1$ \\
\hline
\end{tabular}

*Apoyo Social Afectivo bueno si se obtiene puntuación media mayor de 18.

** Apoyo Social Confidencial bueno si se obtiene puntuación media mayor de 15.

\# Apoyo Social Total bueno si se obtiene puntuación media mayor de 33.

$\&$ Se considera buena función familiar si se obtiene entre 7 y 10 puntos.

del tabaquismo, tan habitual entre estos trabajadores, lo que podría explicar que en estudios como el de Marco et al. ${ }^{19}$ no se evidencie esta relación. Sin embargo, en estudios de base poblacional sí se ha relacionado la exposición laboral a humos y polvos como causa de agudizaciones ${ }^{20-23}$.

El consumo de tabaco es el factor de riesgo fundamental en la patología respiratoria. En diversos estudios ha quedado demostrada la relación entre el consumo de cigarrillos, la génesis de la EPOC y la intensidad de los síntomas respiratorios ${ }^{14,24,25}$. Algunos estudios identifican un consumo de 70 o más paquetes/año como dato específico sugerente de EPOC $^{26}$.

Un elevado porcentaje de pacientes incluidos en el estudio habían sido fumadores $(76 \%)$, superior al encontrado en otros estudios consultados ${ }^{14,19,27}$, con una media de 53,2 paquetes/año, cifra bastante superior a la obtenida en el estudio de Marco et al. ${ }^{19}$ que fue de 24,1 paquetes/año. Cabe resaltar que más de la mitad del total de pacientes $(55,8 \%)$ seguía con el hábito tóxico, y con un aumento del consumo a 61 paquetes/año. Este dato resulta significativo pero es lógico y se justifica porque el índice está en relación con el número de años fumando, por tanto a mayor edad del paciente mayor duración en el tiempo del hábito.

Según los datos recogidos de la bibliografía ${ }^{28}$, hasta un $25 \%$ de los pacientes con EPOC presentan un estado de malnutrición: desnutrición en el paciente enfisematoso avanzado, y obesidad en los enfermos con predominio de bronquitis crónica. Se ha comprobado que el estado nutricional está asociado a la supervivencia de los pacientes con
EPOC, así la malnutrición tiene un efecto claramente negativo en la evolución de la enfermedad, generando una mayor morbilidad y mortalidad ${ }^{7,28,29}$. En la guía clínica para el diagnóstico y tratamiento de la EPOC propuesta por la SEPAR ${ }^{5}$, recientemente publicada, se recomienda una valoración periódica del estado nutricional utilizando el índice de masa corporal (IMC). En nuestro estudio, encontramos un valor medio del IMC de $28,4 \mathrm{~kg} / \mathrm{m}^{2}$, lo que indica sobrepeso, con un $32,2 \%$ de obesos (IMC > 30), frente al bajo peso (IMC<20) que sólo lo presentan el $1,8 \%$.

El 76\% de los pacientes presentaban una o más patologías concomitantes, cifra similar a la encontrada por Ferrer et al. ${ }^{30}$ en un estudio realizado en 321 pacientes varones con EPOC (84\%). A pesar de la alta prevalencia, la comorbilidad suele ser olvidada en los estudios clínicos de los pacientes con EPOC, incluso en los que valoran la calidad de vi$\mathrm{da}^{30-32}$. Sin embargo, se trata de un factor importante pues además de la influencia en la manifestación de síntomas, parece ser el predictor más importante de utilización de servicios sanitarios por parte de estos pacientes ${ }^{33}$.

Al analizar los resultados obtenidos con los cuestionarios de calidad de vida, si consideramos el primero de ellos, NHP, las dimensiones más afectadas fueron las que medían sueño, energía y movilidad. Las puntuaciones fueron superiores en todas las dimensiones a las obtenidas en el trabajo de Alonso et al. ${ }^{9}$, referidas a población general (mayores de 41 años), lo que podríamos explicar por una media de edad más elevada (69,9 años) y una frecuencia alta de patologías asociadas entre los pa- 
cientes del estudio. Si comparamos nuestros resultados con los referidos por García ${ }^{34}$ sobre población diabética e hipertensa de la misma ZBS, observamos que son similares a excepción de la dimensión aislamiento social, que fue más alta en nuestro caso, lo que supone que los pacientes con EPOC perciben mayor aislamiento social que los pacientes con otras patologías.

La comparabilidad de nuestros resultados con otros realizados en pacientes con EPOC en nuestro ámbito, se ve dificultada por varias razones, entre las que destacamos la variabilidad en los cuestionarios utilizados, que son diferentes en cada estudio.

En el estudio realizado por Alonso et al. ${ }^{35}$ en pacientes varones con EPOC estable no dependientes de oxígeno y con seguimiento ambulatorio, utilizando el NHP para valorar la CVRS, destaca un mayor deterioro de la CVRS de éstos respecto a la población general, así como mayor afectación de las dimensiones que valoran sueño, energía y movilidad. Igualmente, otros estudios ${ }^{36}$ en pacientes con EPOC severa demuestran una calidad de vida alterada en todas las dimensiones del NHP.

Con el cuestionario SGRQ la media de puntuación total $(40,9)$ fue más alta que la obtenida por el NHP $(33,4)$, lo que indica peor salud autopercibida por problemas respiratorios. La calidad de vida del paciente con EPOC se vio más afectada en la dimensión que valoraba la actividad (53,8 puntos) y, por tanto, la limitación de ésta por la disnea. Estos resultados coinciden con la mayoría de investigaciones que han utilizado este cuestionario, como los trabajos de Hajiro et al. ${ }^{37}$, Ketelaars et al. ${ }^{38}$ y Perrin et al. ${ }^{39}$, y con otros estudios que afirman que la disnea es el factor que mayor impacto tiene sobre la calidad de vida de los pacientes con EPOC, aun utilizando otros cuestionarios específicos ${ }^{32,40}$ (CRQ) o genéricos ${ }^{41}$ (SIP).

En el estudio realizado por Ferrer et al. ${ }^{30}$, sobre 321 pacientes con EPOC donde utilizaron los mismos cuestionarios para valorar la calidad de vida que en el presente trabajo, se obtuvieron puntuaciones más bajas para todas las dimensiones tanto del NHP como del SGRQ, lo que se traduce en que nuestros pacientes acusan un mayor deterioro en su calidad de vida.

La frecuencia de disfunción familiar encontrada en los pacientes del estudio fue de un $20,3 \%$. No encontramos datos en la bibliografía en los que se haya medido este parámetro en pacientes con EPOC. Si la comparamos con la descrita en otros trabajos sobre población diabética hipertensa es significativamente superior ${ }^{34}$.

Pensamos que las cifras de disfunción familiar encontradas han sido altas, si tenemos en cuenta que factores como la entrevista por un médico desconocido, o en la mayoría de los casos el ir acompañado por un familiar el día de la cita, podría haber infravalora- do esta problemática al generar más reticencias al hablar de la situación familiar emitiendo juicios que forman parte de la esfera íntima y que pueden implicar a terceras personas. En un trabajo realizado por Guyatt et al. ${ }^{40}$ para valorar como se ve afectada la vida diaria por la patología respiratoria en pacientes con EPOC que acudían a una consulta de neumología, observó cierta discrepancia entre la información obtenida de los propios pacientes y la aportada por los cónyuges.

No hemos encontrado en la bibliografía datos referentes a la medida del apoyo social en los pacientes con EPOC, pero si comparamos nuestras cifras con las obtenidas en otros grupos de pacientes con otras patologías crónicas y utilizando el mismo cuestionario $^{11,34}$, observamos que en nuestro caso fueron mayores, sobre todo las referidas a la escala de apoyo afectivo. La población de nuestro estudio reúne algunas de las características descritas en algunos trabajos ${ }^{11}$ que contribuyen a disminuir el apoyo social percibido por el paciente como son la edad elevada, tener una patología crónica, ser analfabeto o carecer de actividad laboral. Sin embargo esto no pareció influir en la percepción de apoyo social en los sujetos del estudio. Por otra parte, no se ha correspondido dicho apoyo social, sobre todo afectivo (frecuencia alta), con los datos obtenidos de disfunción familiar (frecuencia también alta), algo inesperado si consideramos que en nuestra cultura la mayor fuente de apoyo está habitualmente en la familia. El apoyo social y familiar está relacionado con la utilización de servicios sanitarios ${ }^{33}$.

En conclusión, encontramos que los sujetos del estudio poseen un perfil de población anciana, en su mayoría varones, casados, jubilados y con nivel sociocultural bajo. El hábito tabáquico supuso el factor de riesgo más importante a considerar con un consumo más elevado que el encontrado en otros trabajos de ámbito similar. La asociación de comorbilidad es muy frecuente en estos pacientes de edad avanzada, y hemos de considerarla por la implicación en la salud autopercibida y en la necesidad de mayor apoyo socio-familiar. Los aspectos psicosociales influyen de manera importante en la manifestación de síntomas, en la utilización de los recursos sanitarios y pueden suponer una dificultad añadida a la terapia. Existen cuestionarios adaptados a su uso en Atención Primaria, que indagan sobre estos aspectos y aportan la cara oculta de muchas situaciones aparentemente inexplicables, por lo que habría que considerarlos, aun asumiendo la dificultad de su uso.

\section{AGRADECIMIENTOS}

Agradecemos la colaboración desinteresada para el desarrollo de este trabajo a todos los 
miembros de los Centros de Salud Palma-Palmilla y Palo del Distrito Sanitario Málaga, especialmente a Francisco Alcaine y Mercedes Torras, y a todos los pacientes que han participado en el mismo.

\section{CORRESPONDENCIA:}

Francisca Leiva Fernández

Médico de Familia.

C/ Pergolesi n²5 - 29016 Málaga

E-mail: fleiva@hch.sas.cica.es

Telf.: 952307197 - Fax: 952614530

\section{Bibliografía}

1. Morera J. Enfermedad pulmonar obstructiva crónica (EPOC). Magnitud del problema. En: Morera J, editor. Enfermedad pulmonar obstructiva crónica. Barcelona: MCR S.A, 1992; 57-65.

2. Escarrabill J. Situación de oxigenoterapia en nuestro medio. Arch Bronconeumol 1990; 26: 151.

3. Martínez Aragón MN, Llácer A. Mortalidad en España 1997. Mortalidad general y principales causas de muerte y de años potenciales de vida perdidos. Mortalidad por Sida. Boletín Epidemiológico Semanal. Ministerio de Sanidad y Consumo: Instituto de Salud Carlos III. 2000; 8 (23): 253-64.

4. Comité científico del estudio IBERPOC. Proyecto IBERPOC: estudio epidemilógico de la EPOC en España. Arch Bronconeumol 1997; 33: 293-9.

5. Barberá JA, Peces-Barba G, Agustí AGN, Izquierdo JL, Monsó E, Montemayor T, et al. Normativa SEPAR. Guía clínica para el diagnóstico y tratamiento de la enfermedad pulmonar obstructiva crónica. Arch Bronconeumol 2001; 37: 297-316

6. Burrows B, Knudson RJ, Cline M, Lebowizt MD. A reexamination of risk factors for ventilatory impairment. Am Rev Respir Dis 1988; 138: 829-36.

7. White S, Leff A. The relationship of COPD to asthma. In: Chermack NS. ed. Chronic obstructive pulmonary disease. Philadelphia: Ed Saunders Company 1991; 307-16.

8. Celli BR, Snider GL, Heffner J, Tiep B, Ziment I, Make B, et al. American Thoracic Society. Standards for the diagnosis and care of patients with chronic obstructive pulmonary disease. Am J Respir Crit Care Med 1995; 152 (Suppl): $77-120$.

9. Alonso J, Antó JM, Moreno C. Spanich version of Nottingham Health Profile: Translation and peliminary validity. Am J Public Health 1990; 80: 704-8.

10. Ferrer M, Alonso J, Prieto L, Plaza V, Monsó E, Marrades $\mathrm{R}$, et al. Validity and reability of the St. George's Respiratory Questionnaire after adaptation to a different language and culture: the Spanish example. Eur Respir J 1996; 9: 1160-6.

11. Bellón Saameño JA, Delgado Sánchez A, Luna del Castillo JD. Validez y fiabilidad del cuestionario de apoyo social funcional DUKE-UNC-11. Aten primaria 1994; 14 (8): 960.

12. Bellón Saameño JA, Delgado Sánchez A, Luna del Castillo JD. Validez y fiabilidad del cuestionario de función familiar. Apgar familiar. Aten Primaria 1996; 18: 289-96.

13. Jaén A, Ferrer A, Ormaza I, Rué M, Domingo CH, Marín A. Prevalencia de bronquitis crónica, asma y obstrucción al flujo aéreo en una zona urbano-industrial de Cataluña. Arch Bronconeumol 1999; 35: 122-8.

14. Sobradillo Peña V, Miravitlles M, Jimenez CA, Gabriel R, Viejo JL, Masa JF, et al. Estudio IBERPOC en España: prevalencia de síntomas respiratorios habituales y de limitación crónica al flujo aéreo. Arch Bronconeumol 1999; 35: 159-66.

15. Miravitlles M, Murio C, Guerrero T, Segú JL. Tratamiento de la bronquitis crónica y la EPOC en atención primaria. Arch Bronconeumol 1999; 35: 173-8.
16. Baranda García F, Sobradillo Peña V, Talayero Sebastián N, Pérez de las Casas M, Badiola Villa y Ciruelos Ayuso E. Estudio de la concordancia diagnóstica para las patologías asma y EPOC en pacientes ambulatorios. Arch Bronconeumol 1998; 34: 9-13.

17. Villasante Fernández-Montes, en representación del Comité Científico del Estudio IBERPOC. IBERPOC: valoración de resultados. Arch Bronconeumol 1999; 35 (Suppl. 3): 40-3.

18. Oxman AD, Muir DCF, Shanon HS, Stock SR, Hnizdo E, Lange HJ. Occupational dust exposure and chronic obstructive pulmonary disease. A sistematic overview of the evidence. Am Rev Respir Dis 1993; 148: 38-48.

19. Marco Jordán L, Martín Berra JC, Corres Iñigo M, Luque Díez R, Zubillaga Garmendia G. Enfermedad pulmonar obstructiva crónica en la población general. Estudio epidemiológico realizado en Guipúzcoa. Arch Bronconeumol 1998; 34: 23-7.

20. Beckalake MR. Occupational exposures: evidence for a causal association with chronic obstructive pulmonary di sease. Am Rev Respir Dis 1989; 140: 185-91.

21. Burge PS. Occupation and chronic obstructive pulmonary disease (COPD). Eur Respir J 1993; 6: 1095-103.

22. Viegi G, Prediletto R, Paoletti P, Carrozzi L, Fazzi P, Di Pede F, et al. Respiratory effects of occupational exposure in a general population sample in north Italy. Am Rev Respir Dis 1991; 143: 510-5.

23. Xu X, Christiani DC, Dockery DW, Warg L. Exposure-respouse relationship between occupational exposures and chronic illness: a community based study. Am Rev Respir Dis 1992; 146: 413-8.

24. American Thoracic Society. Standards for diagnosis and care of patients wiht chronic obstructive pulmonary disease. Am J Respir Crit Care Med 1995; 152 (Suppl): 77-121.

25. Sánchez Agudo L. Tabaquismo y EPOC. En: Castillo J, ed. EPOC: Perspectivas Actuales. Madrid: Aula Médica Formación Continuada, 1995; 19-24.

26. Badgett RG, Tamaka DJ, Hunt DK, Jelley MJ, Seinberg LE, Steiner JS, et al. Can moderate chronic obstructive pulmonary disease be diagnosed by historcal and physical findings alone? Am J Med 1993; 94 (2): 188-96.

27. Jaén A, Ferrer A, Ormaza I, Domingo CH, Rivero E, Brotones C. Prevalencia de la obstrucción del flujo aéreo y su relación con síntomas respiratorios y tabaquismo en el Vallés Occidental. Arch Bronconeumol 1995; 31: 57.

28. Pascual JM, Carrión F, Sanchez V, Sanchez B, Gonzalez C. Alteraciones nutricionales en pacientes con enfermedad obstructiva crónica avanzada. Med Clin (Barc) 1996; 107: 486-9.

29. Schols AMWJ, Slangen J, Volovics L, Wouters EFM. Weight loss in a reversible factor in the prognosis of chronic obstructive pulmonary disease. Am J Respir Crit Care Med 1998; 157: 1791-7.

30. Ferrer M, Alonso J, Morera J, Marrades RM, Khalaf A, Aguar MC, et al. Chronic Obstructive Pulmonary Disease Stage and Health-Related Quality of Life. Ann Intern Med 1997; 127: 1072-9. 
31. Alonso J, Antó JM, Gonzalez M, Fiz JA, Izquierdo J, Morera J. Measurement of general health status of non-oxygendependent chronic obstructive pulmonary disease patients. Med Care 1992; 30 (Suppl 5): MS 125-35.

32. Elías MT, Ortega F, Sanchéz H, Otero R, Sánchez R. Papel de la disnea en la calidad de vida del paciente con enfermedad pulmonar obstructiva crónica. Arch Bronconeumol 1999; 35: 261-6.

33. Mancera J, Muñoz F, Paniagua F, Fernádez C, Fernádez ML, Blanca FJ. Problemas de salud y factores determinantes del número de visitas a demanda en pacientes hiperutilizadores de un centro de salud. Aten Primaria 2001; 27: 657-62.

34. García AM, Leiva F, Martos F, García A, Prados D, Sánchez de la Cuesta F. Calidad de vida en pacientes con hipertensión y diabetes mellitus tipo 2. Medicina de Familia 2001; 2: 29-34.

35. Alonso J, Antó JM, Gonzalez M, Fiz JA, Izquierdo J, Morera J. Measurement of general health status of non-oxygendependent chronic obstructive pulmonary disease patients. Med Care 1992; 30(Supl 5): MS 125-35.

36. Monsó E, Fiz JM, Izquierdo J, Alonso J, Coll R, Rosell A, et al. Quality of life in severe chronic obstructive pulmonary disease: correlation with lung and muscle function. Respira- tory Medicine 1998; 92: 221-7.

37. Hajiro T, Nishimura K, Tsukino M, Ikeda A, Koyama Izumi T. Analysis of Clinical Methods Used to Evaluate Dysnea in Patients with chronic Obstructive Pulmonary Disease. Am J Respir Crit Care Med. 1998; 158: 1185-9.

38. Ketelaars CA, Schlosser MA, Mostert R, Huyer Abu-Saad $\mathrm{H}$, Halfens RJ, Wonters EF, et al. Determinants of healthrelated quality of life in patients with chronic obstruvtive pulmonary disease. Thorax 1996; Vol 5: 39-45.

39. Perrin C, El Far Y, Vandebos F, Tamiser R, Dumon MC, Lemoigne $\mathrm{F}$, et al. Domiciliary nasl intermittent positive pressure ventilation in severe COPD: effects on lung function and quality of life. Eur Respir J 1997; 10 (12): 2835-9.

40. Ruíz de Oña Lacasta JM, Puente Maestu L, Rodriguez Hermosa JL, Tatay Martí E, Cubillos Marco JM. Comparación de varias escalas de medición para valorar la disnea en las actividades diarias en los pacientes con enfermedad pulmonar obstructiva crónica. Arch Broconeumol 2000; 36: 25-8.

41. Schrier Ac, Dekker FW, Kaptein AA, Dijkman JH. Quality of life in elderly patientes with chronic nonspecific lung disease seen in family practice. Chest 1990; 98: 894-9.

42. Guyatt GH, Townsend M, Berman LB, Pugsley SO. Quality of life in patients with chronic airflow limitation. Br J Dis Chest 1987; 81: 45-54. 